\section{From the authors:}

We are grateful to J. Nasiłowski and colleagues for having pointed out the important problem of the use of noninvasive ventilation (NIV) in Eastern European countries. Unfortunately, we were unaware of the survey conducted in Poland [1], mentioned by the authors, simply because it was not found using any medical search engine (i.e. Pubmed and similar).

We agree with J. Nasiłowski and colleagues that the use of NIV may be different in some geographical locations. However, as stated in the Methods section of our study: "The survey was sent to all members of the Respiratory Intensive Care Assembly from the European Respiratory Society (ERS), members of the Acute Respiratory Failure Group from the European Society of Intensive Care Medicine (ESICM), and physicians working in the emergency department (ED) known to be involved in NIV practice or to have published articles on the topic" [2]. This means that, as pointed out by the authors and as we stated in the Strengths and limitations section of our manuscript, our data cannot be generalised, as we may have missed physicians who are not members of any of these associations. Thus, surveying individuals meeting specific criteria (members of European societies) may have biased the results, but the use of associations' rosters to send out these surveys is common practice for this type of study; therefore there is an a priori bias that could not be easily solved.

Finally, we would like to point out that, as stated in the manuscript's introduction section, the primary aim of our study was to characterise the pattern of NIV utilisation, "placing emphasis on the technical aspects of NIV use" [2] (ventilator and interface type) in different scenarios among European NIV users, rather than identify the prevalence of NIV utilisation in European countries per se.

Concerning the specific points raised by J. Nasilowski and colleagues, we have the following comments.

1) "[The authors] arbitrarily divided Europe geographically into Northern, Central and Southern regions". This division has been employed by several other surveys [3, 4] and is therefore commonly accepted.

2) "[...] only 16 departments/wards (35\% of responders) had been providing NIV to patients with respiratory failure." We do not see a large difference between these data and the results of our survey $(52.9 \%$ of pulmonologists reported $>20 \%$ of patients treated with NIV per yr versus $34.3 \%$ intensivists/ anaesthesiologists and $12.6 \%$ others).
3) "The study [...] represents the extent of NIV use among European leaders in NIV treatment". We respectfully disagree with this point. Being a member of a scientific association does not necessarily mean that you are an expert. About $3 \%$ of our respondents stated that they had no experience with NIV, and the large majority of the intensivists reported treatment of a small percentage of patients (see point 2). Indeed, of the 272 respondents, only a small minority $(\sim 15-20 \%)$ have published at least one paper on the subject, confirming that our survey mainly reflects daily "perceived" practice, rather than an "elite" snapshot.

\section{Crimi*, A. Noto\#, P. Princi ${ }^{\top}$, A. Esquinas ${ }^{+}$and S. Nava ${ }^{\S}$} *Dept of Internal and Specialistic Medicine, Section of Respiratory Diseases, Azienda Ospedaliera Universitaria Policlinico "G. Rodolico", University of Catania, Catania, "Dept of Cardiothoracic and Vascular Anesthesia, Azienda Ospedaliera Universitaria "G. Martino", University of Messina, "National Research Council, Messina, ${ }^{\S}$ Respiratory and Critical Care Unit, Azienda Ospedaliera Universitaria Sant' Orsola-Malpighi, Bologna, Italy, and 'Intensive Care Unit, Hospital Morales Meseguer, Murcia, Spain.

Correspondence: S. Nava, Respiratory and Critical Care Unit, Azienda Ospedaliera Universitaria Sant' Orsola-Malpighi, Via Massarenti no. 9, 40138 Bologna, Italy. E-mail: stefano. nava@aosp.bo.it

Statement of Interest: Statements of interest for P. Princi and S. Nava can be found at www.erj.ersjournals.com/site/misc/ statements.xhtml

\section{REFERENCES}

1 Nasiłowski J, Leszczyk M, Bura M, et al. [A survey of noninvasive ventilation use in respiratory departments in Poland.]. Pneumonol Alergol Pol 2010; 78: Suppl. 1, 43.

2 Crimi C, Noto A, Princi P, et al. A European survey of noninvasive ventilation practices. Eur Respir J 2010; 36: 362-369.

3 Sprung CL, Cohen SL, Sjokvist P, et al. End-of-life practices in European intensive care units: the Ethicus Study. JAMA 2003; 290: 790-797.

4 Nava S, Sturani C, Hartl S, et al. End-of-life decision-making in respiratory intermediate care units: a European survey. Eur Respir J 2007; 30: 156-164.

\title{
Sleep apnoea in Asians and Caucasians: comparing apples and oranges
}

To the Editors:

We read with great interest the recent study in the European Respiratory Journal by YAMAGISHI et al. [1]. The study sheds new light on our understanding of ethnic differences in susceptibility to obstructive sleep apnoea (OSA). Previous studies have shown consistently that among patients referred to sleep laboratories, Asians present a higher apnoea/hypopnoea index (AHI) than Caucasians matched for age and body mass index (BMI). Conversely, when matched for AHI, Asians have a lower BMI 
than Caucasians. These observations have lead to the concept that Asians are at increased risk of OSA [2]. One attractive explanation is that Asians have decreased cranial base dimensions that would render the upper airways more prone to collapse. However, if Asians were more prone to OSA, one would predict a higher prevalence of OSA among Asians compared with Caucasians. In contrast, due to cultural and dietary aspects, Asians would be protected from OSA on the basis that obesity is less common than among white Americans. Previous independent epidemiological studies from Asia and the USA showed similar prevalence of OSA in these populations [3, 4]. YAMAGISHI et al. [1] took a step forward and directly compared the prevalence of OSA among Hispanic and white Americans (from the Minnesota field centre, St Paul, MN, USA) with the prevalence of OSA drawn from three community-based cohorts in Japan. The strength of this study was that the authors used identical procedures to detect sleepdisordered breathing in the US and Japanese populations. The authors showed that the prevalence of OSA in the Japanese population was lower than in the US population. The lower prevalence of OSA in Asians was explained by the fact that the Japanese had a lower BMI. The authors concluded that the majority of the race/ethnic differences in OSA prevalence were explained by differences in BMI among populations.

We would like to add to this discussion the concept that comparing BMI between Asians and Caucasians is not appropriate. This concept is not new and the World Health Organization (WHO) defined overweight and obesity among Asians with BMI cut-offs (23 and $25 \mathrm{~kg} \cdot \mathrm{m}^{-2}$, respectively) that are therefore lower than the cut-offs used for Caucasians (25 and $30 \mathrm{~kg} \cdot \mathrm{m}^{-2}$, respectively). These guidelines reflect the knowledge that Asians are at increased cardiovascular risk at lower BMI. The reason is well established and Asians have proportionally more fat for a similar BMI compared with Caucasians. São Paulo, Brazil, has the largest Japanese community outside Japan and therefore provided us the opportunity to observe that Japanese-Brazilians referred for polysomnography had a lower BMI than Caucasians with a similar severity of OSA. Multiple regression showed that being Japanese, increasing age, BMI, neck circumference and sleepiness were independently associated with OSA. This observation would lead to the impression that Japanese were at higher risk of OSA. However, when the WHO criteria for obesity was taken into consideration, obesity was actually more common among Asians than Caucasians and being Asian was no longer a risk factor for OSA on multiple regression [5]. We conclude that more studies that consider both craniofacial anatomy and body composition (rather than BMI) and compare Asians and Caucasians are necessary to better understand the ethnic differences in OSA presentation. These studies will also improve our understanding of the pathophysiology of the disease. Meanwhile, all authors should remember that the absolute BMI level is not a good surrogate marker of obesity when Asians and Caucasians are compared.

\section{P.R. Genta and G. Lorenzi-Filho}

Pulmonary Division, Heart Institute (InCor), University of São Paulo School of Medicine, São Paulo, Brazil.

Correspondence: P.R. Genta, Av. Dr. Enéas de Carvalho Aguiar, 44-7th floor, 05403-900, São Paulo, Brazil. E-mail: prgenta@usp.br

Statement of Interest: None declared.

\section{REFERENCES}

1 Yamagishi K, Ohira T, Nakano H, et al. Cross-cultural comparison of the sleep-disordered breathing prevalence among Americans and Japanese. Eur Respir J 2010; 36: 379-384.

2 Ong K, Clerk A. Comparison of the severity of sleep-disordered breathing in Asian and Caucasian patients seen at a sleep disorders center. Respir Med 1998; 92: 843-848.

3 Ip M, Lam B, Lauder I, et al. A community study of sleep-disordered breathing in middle-aged Chinese men in Hong Kong. Chest 2001; 119:62-69.

4 Young T, Palta M, Dempsey J, et al. The occurrence of sleep-disordered breathing among middle-aged adults. N Eng J Med 1993; 328: 1230-1235.

5 Genta PR, Marcondes B, Danzi N, et al. Ethnicity as a risk factor for obstructive sleep apnea: comparison of Japanese descendants and white males in São Paulo, Brazil. Braz J Med Biol Res 2008; 41: 728-733.

DOI: $10.1183 / 09031936.00200510$

From the authors:

We thank P.R. Genta and G. Lorenzi-Filho for their interest in and comments on our study [1]. One of our major findings was that the prevalence of sleep-disordered breathing (SDB) was similar among the community-based samples of Caucasians, Hispanics and Japanese within the same body mass index (BMI) stratum. However, a major point of the critique from P.R. Genta and G. Lorenzi-Filho was that ethnic differences in body fat composition need to be considered when comparing the prevalence or severity of SDB. Based on their clinical findings [2], they suggested using different cut-offs for defining obesity (i.e. $\geqslant 30 \mathrm{~kg} \cdot \mathrm{m}^{-2}$ for Caucasians and $\geqslant 25 \mathrm{~kg} \cdot \mathrm{m}^{-2}$ for Asians).

In response to P.R. Genta and G. Lorenzi-Filho we recalculated the prevalence of SDB by ethnicity using their recommended dichotomous obesity cut-offs. The SDB prevalence remained higher among Americans than Japanese regardless of obesity stratum. In the re-defined obese stratum, SDB prevalence was $21 \%$ for Japanese, $40 \%$ for Hispanics ( $p<0.001$ for difference from Japanese) and $44 \%$ for white Americans $(\mathrm{p}<0.001)$; and in the non-obese stratum, $17 \%$ for Japanese, $32 \%$ for Hispanics $(p=0.001)$ and $27 \%$ for Whites $(p=0.01)$. These findings do not support their hypothesis that Japanese have higher SDB prevalence within these new obesity categories.

We agree that BMI may not be the best body composition marker to compare ethnicities; however, no better markers than BMI have been reported for the general population. Further cross-cultural studies in community settings should be performed to address this issue.

\section{K. Yamagishi*, H. Iso", A. Kitamura , A.R. Folsom ${ }^{+}$and T. Tanigawa ${ }^{\S}$}

*Dept of Public Health Medicine, Graduate School of Comprehensive Human Sciences and Institute of Community Medicine, University of Tsukuba, Tsukuba, "Public Health, Dept of Social and Environmental Medicine, Osaka University Graduate School of Medicine, Suita, "Osaka Medical Center for Health Science and Promotion, Osaka, ${ }^{\S}$ Dept of Public Health, 